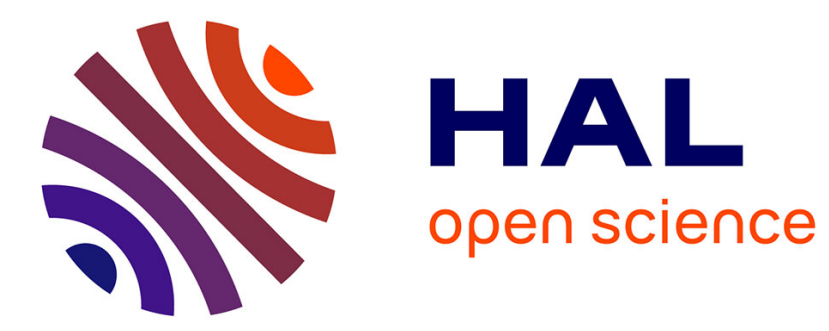

\title{
First-principles study on the role of silicon point defects on PERC solar cell degradation
}

Elisa Tejeda-Zacarias, Philippe Baranek, Holger Vach

\section{To cite this version:}

Elisa Tejeda-Zacarias, Philippe Baranek, Holger Vach. First-principles study on the role of silicon point defects on PERC solar cell degradation. 2021 IEEE 48th Photovoltaic Specialists Conference (PVSC), Jun 2021, Fort Lauderdale, United States. pp.0809-0813, 10.1109/PVSC43889.2021.9518589 . hal03475970

\section{HAL Id: hal-03475970 \\ https://hal.science/hal-03475970}

Submitted on 11 Dec 2021

HAL is a multi-disciplinary open access archive for the deposit and dissemination of scientific research documents, whether they are published or not. The documents may come from teaching and research institutions in France or abroad, or from public or private research centers.
L'archive ouverte pluridisciplinaire HAL, est destinée au dépôt et à la diffusion de documents scientifiques de niveau recherche, publiés ou non, émanant des établissements d'enseignement et de recherche français ou étrangers, des laboratoires publics ou privés. 


\title{
First-principles study on the role of silicon point defects on PERC solar cell degradation
}

\author{
Elisa Tejeda-Zacarias ${ }^{1,2}$, Philippe Baranek ${ }^{1,2}$, and Holger Vach ${ }^{3}$ \\ ${ }^{1}$ EDF R\&D, Department EFESE, EDF Lab Paris-Saclay, 7 boulevard Gaspard Monge, F-91120 Palaiseau, France \\ ${ }^{2}$ IPVF, 18 boulevard Thomas Gobert, F-91120 Palaiseau, France \\ ${ }^{3}$ LPICM, CNRS, Ecole Polytechnique, IP de Paris, F-91128 Palaiseau Cedex, France
}

\begin{abstract}
One of the causes of decrease in the performance of silicon-based solar pannels is linked to light and elevated temperature induced degradation (LeTID). Even if experimental evidence shows that different defects in the bulk material of the modules are involved in the mechanisms behind LeTID, its origins remain unresolved. First-principles methods result in powerful tools to understand this degradation at the nano and microscopic levels. In the present work we propose an approach to model LeTID precursors mechanisms by using ab initio methodology.

Index Terms-LeTID, ab initio, hydrogen, interstitial defects, silicon, PERC modules
\end{abstract}

\section{INTRODUCTION}

Among silicon-based solar panels, Passivated Emitter and Rear Cell (PERC) modules represent a recent and promising technology due to their exceptional efficiency of $24 \%$ [1]. Nevertheless, a decrease of their performance associated with the Light and elevated Temperature Induced Degradation (LeTID) is observed after some years of use. The underlying mechanisms of this complex phenomena remains unclear [2].

Several models have been proposed based mainly on experimental results. LeTID, characterized by degradation and recovery of performances, has been generally explained by simple models that consider three main phases [3]-[6] :

1) Defect formation: Formation of a precursor recombination inactive defect that causes the degradation. The bulk material contains impurities coming from its synthesis. After the firing step, hydrogen and other impurities like metals, might be released into the bulk material of the wafer.

2) Degradation: Decrease of the charge carriers lifetimes until a minimum value when the module is exposed to environmental conditions.

3) Recovery: The degraded state, which is metastable, evolves toward a recovered stable state in which the recombination defect is passivated.

These simple models are able to explain the main behaviors of LeTID such as its dependence on the peak firing temperature and the cooling rate [7] and the formation of a bulk defect in the wafer prior to degradation [8]. But they do not allow to have a precise description of the role of silicon intrinsic and extrinsic defects on the LeTID precursor mechanisms.

Nowadays, from an experimental point of view, there is a general consensus around the fundamental role of hydrogen as precursor for LeTID [6], [9]-[14]. There is evidence that metals, like cobalt and nickel, coming from the firing step into the bulk silicon, play also a role as LeTID precursors [10], [11]. Other studies have shown a dependence between LeTID and interstitial silicon atoms and lattice vacancies [10]. The similarities of the LeTID cycle in different types of siliconbased modules suggest that the defects precursors along these materials are the same [15] but not in a conclusive way. At the microscopic scale, a study of defects in crystalline silicon and its evolution under temperature changes and light exposure is expected to reveal this problem in a much more complete form.

In this context, in order to analyze and rationalize the behavior of different defects proposed as degradation precursors, theoretical atomic approaches, such as density functional theory (DFT) and molecular dynamics, result in powerful tools to understand the degradation mechanisms. Even if hydrogen defects, some metallic impurities, and self-defects in silicon have been studied before using a first principle approach [16], [17], a first-principles multiscale study to understand the complete LeTID mechanisms has not been presented so far.

\section{Methodology}

Figure 1 shows the three steps LeTID model considered here. The first step concerns the study of the stability and electronic properties of active defects in the bulk or at the surfaces

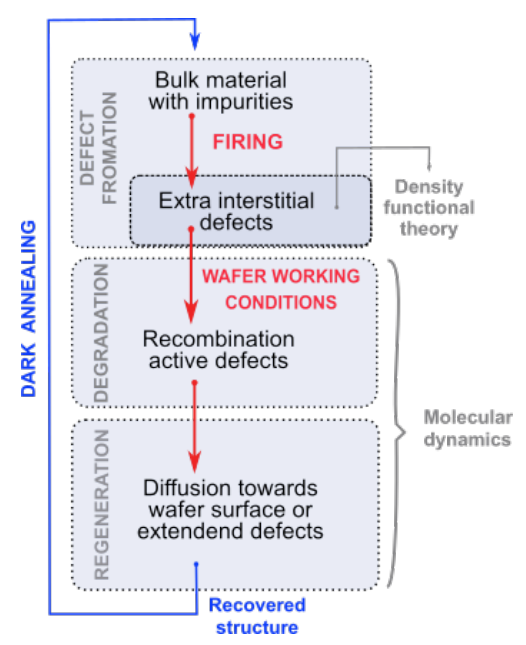

Fig. 1. Methodology proposed for this work. 
of crystalline silicon. The choices of defects, crystalline bulk and slab of silicon are based on the various assumptions of a common defect causing LeTID in different silicon based wafers [15] and the existence of denuded zones in multicrystalline silicon [10] which can passivate the defect.

In this work, we focus at the first-principles level on the behavior of interstitial hydrogen defects in section III, and selfsilicon defects (such as interstitial silicon $S i_{i}$ and vacancies $V$ ) in section IV. Defects including metals, along with metalhydrogen complexes, will be the subject of a forthcoming work. This work represents a first study of such structures by the use DFT. Later on, molecular dynamics simulations will be performed to investigate the temperature and carrier injection effects and reproduce the degradation and regeneration processes.

The DFT defective structures were simulated using the code CRYSTAL17 [18]. In order to reproduce the experimental value of the indirect band gap of silicon, the hybrid functional DFT-PBEsol [19] was optimized with a percentage of Hartree Fock $(\mathrm{HF})$ contribution of $12.5 \%$. The obtained electronic and structural properties of crystalline silicon such as cell parameter, $\Gamma$-phonon frequency, dielectric constant and indirect band gap are presented in table I. The hybrid functional optimization leads to a good representation of these properties with respect to experiments. Even more, figure 2 shows a good agreement between the electronic band structure computed with respect to experimental data [20].

For the defective structures, a supercell approach was employed. Relaxation of the host atoms around the impurity was also allowed by minimizing the energy with respect to the atoms positions and cell deformations. Then, a phonon frequency calculation was performed in order to check the dynamical stability of presumably equilibrium structure.

Since in this work the defects are considered as isolated, further sizes of supercell were explored to determine the convergence of the formation energy with respect to the number of atoms for properly computing the thermodynamic properties of the systems. Likewise, the band gap convergence was achieved to accurately obtain the electrical properties. The difference between bandgap values for two supercells of different sizes has been fixed at $10^{-2} \mathrm{eV}$ to be considered as converged.

An analysis of the defects formation energies is performed in order to study the stability of different interstitial hydrogen impurities. Such formation energies are computed according to the following reaction:

TABLE I

CALCUlated CEll PARAMETER $(a), \Gamma$-PHONON FREQUENCY $(\omega)$, DIELECTRIC CONSTANT $(\epsilon)$ AND INDIRECT BAND GAP $\left(E_{g_{i}}\right)$ OF SILICON. THE EXPERIMENTAL DATA ARE GIVEN FOR COMPARISON.

\begin{tabular}{|c|c|c|c|c|}
\cline { 2 - 5 } \multicolumn{1}{c|}{} & $\begin{array}{c}a \\
{[\stackrel{A}{c}]}\end{array}$ & $\begin{array}{c}\omega \\
{\left[\mathrm{cm}^{-1}\right]}\end{array}$ & $\epsilon$ & $\begin{array}{c}E_{g_{i}} \\
{[\mathbf{e V}]}\end{array}$ \\
\hline PBEsol_12.5 & 5.423 & 535 & 10.7 & 1.17 \\
Experimental & 5.431 & 520 & 11.4 & 1.17 \\
error in \% & 0.14 & 2.80 & 6.14 & 0.00 \\
\hline
\end{tabular}

$$
\text { Crystal }+ \text { React }_{A} \rightarrow \text { Defective } \text { React }_{B}
$$

From where the formation energy is defined as:

$$
\Delta E=E_{D e f}+E_{R_{B}}-E_{C r y s t}-E_{R_{A}}
$$

Assuming that the LeTID precursors might act as a trap of photo-generated carriers, they might give rise of a number of levels lying within the silicon band gap. Therefore, different charge states of the same defect are studied to observe their influence in the electronic properties of silicon. It is possible to define the thermodynamic ionization level (TIL) as the crossing of different defect formation energies of different charge states of the same defect.

\section{INTERSTITIAL HYDROGEN IN CRYSTALLINE SILICON}

The study of monoatomic and diatomic interstitial hydrogen defects is presented in this section. The DFT study includes an analysis of their stability regarding their formation energy, and their influence in the band structure of crystalline silicon.

\section{A. Monoatomic hydrogen defects}

The stable position for interstitial monoatomic defects are presented in figure 3 . Hydrogen in the positive charge state $\left(H^{+}\right)$seeks the regions of highest electron charge density (Fig. 3a). The neutral interstitial hydrogen $H^{0}$ is obtained to be at the midway of two silicon atoms, also called bond center (BC), (Fig. 3b). On the other hand, the negative charge state $H^{-}$is found to reside in the vicinity of the lower electron density, at the tethraedral interstitial site $\left(T_{d}\right)$ (Fig. 3c). The presence of $\mathrm{H}^{+}$introduces a level in the band gap which is empty for the case of the positive charge state but will be occupied for the neutral charge state.

The relative stability of the impurities is evaluated by analyzing their formation energies for converged supercells with 216 atoms. The converged formation energies and band gap values for the defective structures are presented in table II.

The chemical potential $\mu$ is defined as the reservoir with which the defects exchange electrons. As observed in figure 4 , the donor level $E_{D}$ is defined as the Fermi-level position at

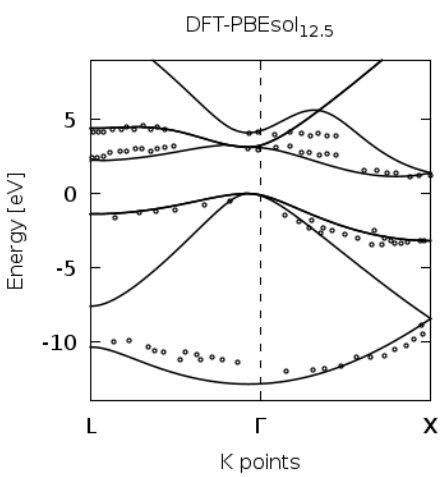

Fig. 2. Electronic band structure computed via the PBEsol 12.5 functional. The experimental data [20] (dots) are given for comparison. 


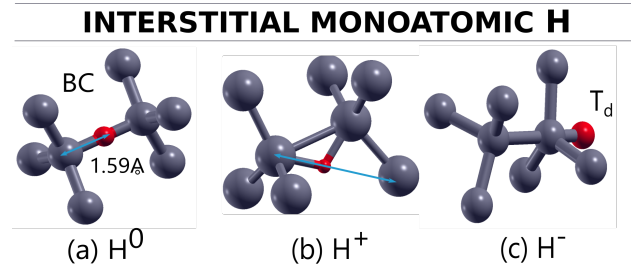

INTERSTITIAL H DIMERS

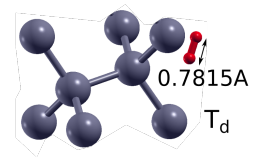

(d) $\mathrm{H}_{2}$ (e) $\mathrm{H}_{2} *$

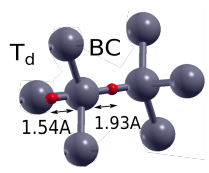

Fig. 3. Minium energy atomic configurations of interstitial $\mathrm{H}, \mathrm{H}_{2}$ and $\mathrm{H}_{2}^{*}$ hydrogen in bulk Si.

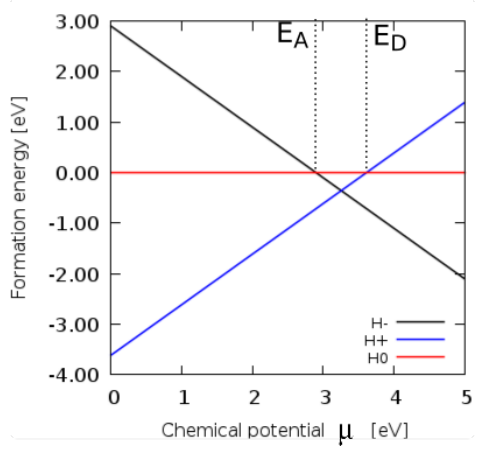

Fig. 4. Relative formation energies for $\mathrm{H}$ charge states as a function of the chemical potential $\mu$.The acceptor and donor levels are indicated as $E_{A}$ and $E_{D}$, respectively for a converged supercell with 216 atoms.

which $H^{+}$and $H^{0}$ have equal formation energies. Likewise, the acceptor level $E_{A}$ is defined as the Fermi-level position at which $H^{0}$ and $H^{-}$formation energies agree. Such energies are located at 2.89 and $3.60 \mathrm{eV}$, respectively and define the Fermi energy range where each charged state is stable. It is observed that the neutral charge state, $H^{0}$, is never thermodynamically stable. Then, p-doped and n-doped materials will favor the presence of $\mathrm{H}^{-}$and $\mathrm{H}^{+}$defects, respectively. As p-doped samples have shown a higher extent of LeTID, it privileges the study of $H^{-}$with respec to to other monoatomic hydrogen defects concerning LeTID.

Figure 5 presents a comparison of the band structure of crystalline silicon and defective structures containing the three charge states of interstitial monoatomic hydrogen. Such structures were obtained for supercells with 216 silicon atoms, for which the electronic properties of the structures are converged. The defective structures containing $\mathrm{H}^{+}$and $\mathrm{H}^{-}$generate a state that is close to the band edges (conduction band and valence band respectively) so they correspond to shallow traps. In the case of the neitral hydrogen charge state, the generated energy level is close to the middle of band gap, so it corresponds to a deep trap.

\section{B. Interstitial hydrogen molecules}

Theory and experiment have confirmed the presence of two stable forms of diatomic hydrogen in crystalline silicon [16], [21]: the $\mathrm{H}_{2}$ as a bounded pair molecule in the tetrahedral interstitial site $\left(T_{d}\right)$, presented in Fig. 3(d), and, the $H_{2}^{*}$ complex consisting in one hydrogen atom in a bond-centerlike position and the other in an antibonding-type position (see Fig. 3e).

The complex has $C_{3 v}$ symmetry and consists of one $\mathrm{H}$ in a bond-center-like position and one $\mathrm{H}$ in an antibondingtype position. The $\mathrm{Si}$ atom in between the two hydrogens is substantially relaxed from its equilibrium site, breaking the original Si-Si bond; the resulting dangling bond is tied off by the bond-center-type hydrogen. Even though the $\mathrm{H}_{2} *$ complex has been computed to be slightly higher in energy than are $\mathrm{H}_{2}$ molecules, it has been proposed to play a role in diffusion, because its activation barrier for migration is lower than that of molecular hydrogen [21]. If this is the case, this type of hydrogen defect might play a role in diffusion and therefore, has a greater impact in LeTID.

From figure 5, both $\mathrm{H}_{2}^{*}$ and $\mathrm{H}_{2}$ defects affect similarly the silicon band structure, generating an energy level near the balance band. The difference in the band gap in both defective structures is within the numerical error of the band gap value.

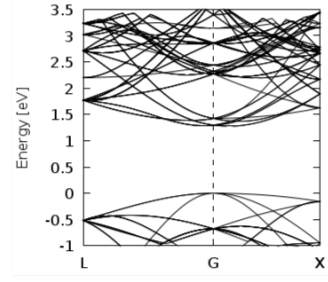

(a) Crystal

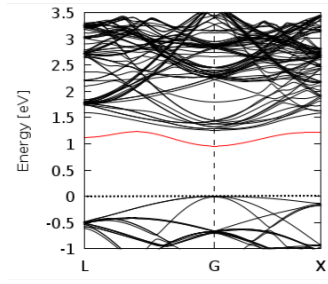

(d) $\mathrm{H}^{+}$

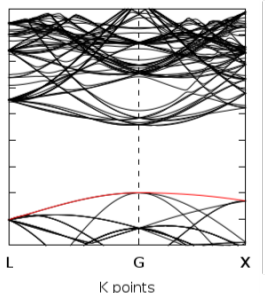

(b) $\mathrm{H}_{2}$

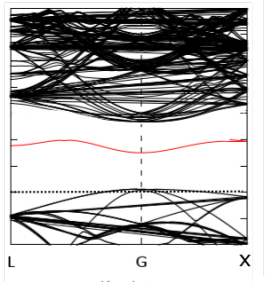

(e) $\mathrm{H}^{0}$

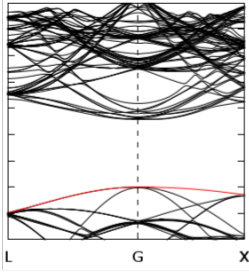

(c) $\mathrm{H}_{2}{ }^{*}$

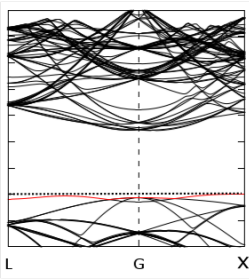

(f) $\mathrm{H}^{-}$
Fig. 5. Band structure of crystalline silicon and defective structures containing interstitial hydrogen defects. Supercell with 216 silicon atoms.

\begin{tabular}{|c|c|c|}
\hline Defect & $E_{\text {form }}[\mathbf{e V}]$ & Band Gap [eV] \\
\hline$H^{+}$ & -1.79 & 0.96 \\
$H^{0}$ & 1.83 & 1.17 \\
$H^{-}$ & 4.78 & 1.09 \\
$H_{2}$ & 1.08 & 1.18 \\
$H_{2}^{*}$ & 1.39 & 1.18 \\
\hline \multicolumn{3}{|c}{ TABLE II }
\end{tabular}

RELATIVE FORMATION ENERGIES AND BAND GAP FOR INTERSTITIAL SILICON ATOMS. 


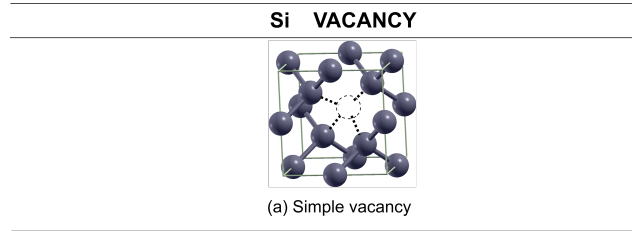

(a) Simple vacancy

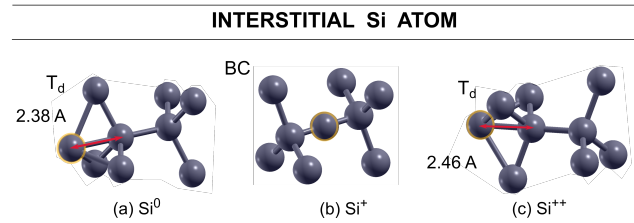

Fig. 6. Minium energy atomic configurations of self silicon defects.

\begin{tabular}{|c|c|c|}
\hline Defect & $E_{\text {form }}[\mathbf{e V}]$ & Band Gap [eV] \\
\hline$S i_{i}^{++}$ & -3.14 & 0.73 \\
$S i_{i}^{0}$ & 4.45 & 1.12 \\
\hline
\end{tabular}

RELATIVE FORMATION ENERGIES AND BAND GAP FOR INTERSTITIAL SILICON ATOMS.

\section{SELF DEFECTS IN CRYSTALLINE SILICON}

\section{A. Interstitial silicon defects}

The most common charged states of interstitial silicon are $S i_{i}^{0}, S i_{i}^{+}$and $S i_{i}^{++}$. Simulations show that the neutral charge state of interstitial silicon $S i_{i}^{0}$ is found to be at $T_{d}$ position, while literature indicates that it sits in a split- $j 110_{i}$ configuration where it shares a lattice site with another silicon atom. The double ionized charge state of interstitial silicon $S i_{i}^{++}$is found to be at $T_{d}$ position as well and, the ionized state $S i_{i}^{+}$, sits in a bond-centered position, both in agreement with the literature.

The converged formation energies and the band gap values for these defects are presented in table III. For the case of $S i_{i}^{+}$, the structure behaves as a metal like material (no band gap), so the formation energy is not computed. and the band diagram is not presented in figure 7.

From figure 7 it is observed that the interstitial $S i_{i}^{0}$ is a deep energy level defect, while $\mathrm{Si}_{i}^{++}$generates an energy level near the conduction band, corresponding to a shallow defect.

\section{B. Vacancies}

Previous ab initio simulations and experimental observations show that the most stable geometries of the neutral and

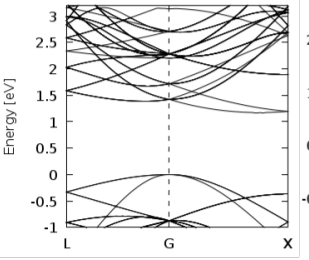

(a) Crystal

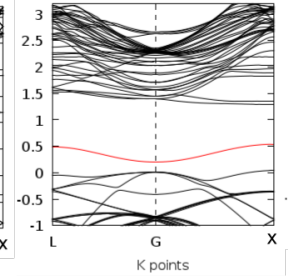

(b) $\mathrm{Si}_{\mathrm{i}}$

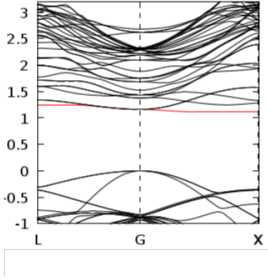

(c) $\mathrm{Si}^{++}$
Fig. 7. Band structure of crystalline silicon and defective structures containing interstitial hydrogen defects. Supercell with 216 silicon atoms. charge states of the silicon vacancy $V^{0}$ has a $D_{2 d}$ symmetry [22], as shown in figure 3. Some other charged states of the silicon vacancy, such as $V^{++}, V^{+}, V^{--}$and $V^{-}$remain under study.

\section{CONCLuSions}

A DFT hybrid functional approach has been used to spot the most likely LeTID precursor. Considering crystalline silicon as bulk material, hydrogen and self-silicon defects were studied. The obtained results are in good agreement with other theoretical and experimental data proving that this approach allow us to obtain a qualitative and quantitative description of these kinds of defects.

All of the defects studied in this work, except for $S i_{i}$ and $H_{0}$, correspond to shallow defects in silicon. This type of defects is characterized by having an ionisation energy of the order of kT and they generally act as dopants. On the other hand, the neutral charge states of hydrogen and silicon are deep level defects that might act as recombination centers. Nevertheless, this behavior might differ under charge induced degradation conditions.

This study shows that among interstitial hydrogen defects, $H^{-}$and $H_{2}^{*}$ are more likely to play a role in LeTID. Structures like double vacancies, defects with metallic impurities and metal-hydrogen complexes remain under study. Nevertheless, such results encourage us to further analyze these more complex defective structures.

Based on these results, the degradation and recovery phases will be treated with molecular dynamics in order to include temperature evolution and carrier injection. By comparing the obtained results with quantities as charge carrier lifetimes, capture cross-section ratio, binding energies upon thermal or light dissociation, and the spatial distribution of the degradation, the role of the simulated defects in LeTID will be validated in forthcoming work.

\section{REFERENCES}

[1] R. Chen, H. Tong, H. Zhu, C. Ding, H. Li, D. Chen, B. Hallam, C. M. Chong, S. Wenham, and A. Ciesla, " $23.83 \%$ efficient monoperc incorporating advanced hydrogenation," Progress in Photovoltaics: Research and Applications, vol. 28, pp. 1239-1247, 2020.

[2] K. Ramspeck, S. Zimmermann, H. Nagel, A. Metz, Y. Gassenbauer, B. Birkmann, and A. Seidi, "Light induced degradation of rear passivated mc-si solar cells," Proc. 27th Eur. Photovolt. Sol. Energy Conf., vol. 2DO.3.4, p. 861-865, 2012.

[3] D. Sperber, A. Herguth, and G. Hahn, "A 3-state defect model for lightinduced degradation in boron-doped float-zone silicon," physica status solidi (RRL) - Rapid Research Letters, vol. 11, no. 3, p. 1600408, 2017.

[4] K. Krauß, A. A. Brand, F. Fertig, S. Rein, and J. Nekarda, "Fast regeneration processes to avoid light-induced degradation in multicrystalline silicon solar cells," IEEE Journal of Photovoltaics, vol. 6, no. 6, pp. 1427-1431, 2016.

[5] W. Kwapil, T. Niewelt, and M. Schubert, "Kinetics of carrier-induced degradation at elevated temperature in multicrystalline silicon solar cells," Solar Energy Materials and Solar Cells, vol. 173, 062017.

[6] A. Ciesla, S. Wenham, R. Chen, C. Chan, D. Chen, B. Hallam, D. Payne, T. Fung, M. Kim, S. Liu, S. Wang, K. Kim, A. Samadi, C. Sen, C. Vargas Castrillon, U. Varshney, B. Vicari Stefani, P. Hamer, G. Bourret-Sicotte, and M. Abbott, "Hydrogen-induced degradation," pp. 0001-0008, 062018. 
[7] H. C. Sio, H. Wang, Q. Wang, C. Sun, W. Chen, H. Jin, and D. Macdonald, "Light and elevated temperature induced degradation in p-type and n-type cast-grown multicrystalline and mono-like silicon," Solar Energy Materials and Solar Cells, vol. 182, pp. 98-104, 082018.

[8] D. Sperber, F. Furtwängler, A. Herguth, and G. Hahn, "Does letid occur in c-si even without a firing step?," AIP Conference Proceedings, vol. 2147, no. 1, p. 140011, 2019.

[9] H. C. Sio, D. Kang, X. Zhang, J. Yang, J. Jin, and D. Macdonald, "The role of dark annealing in light and elevated temperature induced degradation in p-type mono-like silicon," IEEE Journal of Photovoltaics, vol. 10, no. 4, pp. 992-1000, 2020.

[10] T. Niewelt, F. Schindler, W. Kwapil, R. Eberle, J. Schön, and M. C. Schubert, "Understanding the light-induced degradation at elevated temperatures: Similarities between multicrystalline and floatzone p-type silicon," Progress in Photovoltaics: Research and Applications, vol. 26, no. 8, pp. 533-542, 2018.

[11] J. Schmidt, D. Bredemeier, and D. C. Walter, "On the defect physics behind light and elevated temperature-induced degradation (letid) of multicrystalline silicon solar cells," IEEE Journal of Photovoltaics, vol. 9, no. 6, pp. 1497-1503, 2019.

[12] T. H. Fung, M. Kim, D. Chen, A. Samadi, C. E. Chan, B. J. Hallam, S. Wenham, and M. Abbott, "Influence of bound hydrogen states on carrier-induced degradation in multi-crystalline silicon," AIP Conference Proceedings, vol. 1999, no. 1, p. 130004, 2018.

[13] A. Zuschlag, D. Skorka, and G. Hahn, "Degradation and regeneration in mc-si after different gettering steps," Progress in Photovoltaics: Research and Applications, vol. 25, no. 7, pp. 545-552, 2017.

[14] M. A. Jensen, A. Zuschlag, S. Wieghold, D. Skorka, A. E. Morishige, G. Hahn, and T. Buonassisi, "Evaluating root cause: The distinct roles of hydrogen and firing in activating light- and elevated temperature-induced degradation," Journal of Applied Physics, vol. 124, no. 8, p. 085701 , 2018.

[15] D. Chen, M. Vaqueiro Contreras, A. Ciesla, P. Hamer, B. Hallam, M. Abbott, and C. Chan, "Progress in the understanding of lightand elevated temperature-induced degradation in silicon solar cells: A review," Progress in Photovoltaics: Research and Applications, pp. 122, 2020.

[16] C. Herring, N. M. Johnson, and C. G. Van de Walle, "Energy levels of isolated interstitial hydrogen in silicon," Phys. Rev. B, vol. 64, p. 125209, Sep 2001.

[17] Z. Ibrahim, A. Shkrebtii / Chkrebtii, F. Gaspari, Z.-D. I. F., I. Kupchak, and D. Korbutyak, "Characterization of hydrogen in c-si and a-si:h from ab-initio molecular dynamics: Structure, optics and vibrations," 29th European Photovoltaic Solar Energy Conference and Exhibition, vol. 1BV.6.26, pp. 127 - 130, 2020.

[18] R. Dovesi, A. Erba, R. Orlando, C. M. Zicovich-Wilson, B. Civalleri, L. Maschio, M. Rérat, S. Casassa, J. Baima, S. Salustro, and B. Kirtman, "Quantum-mechanical condensed matter simulations with crystal," WIREs Computational Molecular Science, vol. 8, no. 4, p. e1360, 2018.

[19] J. P. Perdew, A. Ruzsinszky, G. I. Csonka, O. A. Vydrov, G. E. Scuseria, L. A. Constantin, X. Zhou, and K. Burke, "Restoring the density-gradient expansion for exchange in solids and surfaces," Phys. Rev. Lett., vol. 100, p. 136406, Apr 2008.

[20] J. E. Ortega and F. J. Himpsel, "Inverse-photoemission study of ge(100), si(100), and gaas(100): Bulk bands and surface states," Phys. Rev. B, vol. 47, pp. 2130-2137, Jan 1993.

[21] C. G. Van de Walle and J. Neugebauer, "Hydrogen in semiconductors," Annual Review of Materials Research, vol. 36, no. 1, pp. 179-198, 2006.

[22] M. J. Puska, S. Pöykkö, M. Pesola, and R. M. Nieminen, "Convergence of supercell calculations for point defects in semiconductors: Vacancy in silicon,” Phys. Rev. B, vol. 58, pp. 1318-1325, Jul 1998. 